\title{
Manifestaciones gastrointestinales en neurofibromatosis tipo 1. Reporte de un caso
}

\author{
Daniela Méndez, ${ }^{1}$ Martin Oricchio, ${ }^{1}$ Yesica Pontet, ${ }^{1}$ Martha Otero, ${ }^{2}$ Felipe Muñiz, ${ }^{2}$ Santiago \\ Cubas, ${ }^{3}$ Nicolas Gonzalez ${ }^{1}$
}

\footnotetext{
${ }^{1}$ Clinica de Gastroenterología, Hospital de Clinicas "Dr. Manuel Quintela".

${ }^{2}$ Cátedra y departamento de Anatomia Patológica, Hospital de Clínicas "Dr. Manuel Quintela".

${ }^{3}$ Clinica Quirurgica B, Hospital de Clínicas "Dr. Manuel Quintela".

Montevideo, Uruguay.
}

Acta Gastroenterol Latinoam 2020;50(4):474-478

Recibido: 28/02/2020 / Aceptado: 11/09/2020 / Publicado online: 14/12/2020

\section{Resumen}

La neurofibromatosis tipo 1, o enfermedad de von Recklinghausen, es un desorden neurocutáneo hereditario con compromiso gastrointestinal en el 5-25\% de los pacientes, el cual se presenta posterior a las manifestaciones cutáneas. Sólo 5\% presenta sintomas como dolor abdominal, diarrea, masa palpable, sangrado, obstrucción o perforación intestinal. Presenta riesgo incrementado de desarrollar neoplasias digestivas, las cuales se ubican con mayor frecuencia en el intestino delgado. Se presenta el caso de un paciente con el antecedente de neurofibromatosis tipo 1, que en el contexto

Correspondencia: Daniela de los Ángeles Méndez Loayza Avenida Italia S/N, Hospital de Clinicas, piso 4. Montevideo, Uruguay Fax.:+598-2-4808472 / Cel.: 092131331

Correo electrónico: danieladelosangelesmd@gmail.com de una hemorragia digestiva se diagnostica un tumor del estroma gastrointestinal yeyunal y una lesión polipoidea cecal que corresponde a un pólipo fibroide inflamatorio.

Palabras claves. Neurofibromatosis tipo 1, tumor del estroma gastrointestinal, pólipo fibroide inflamatorio.

\section{Gastrointestinal Manifestations in Type 1 Neurofibromatosis. A Case Report}

\section{Summary}

Neurofibromatosis type 1, also known as von Recklinghausen disease, is an inherited neurocutaneous disorder with gastrointestinal involvement in 5-25\% of the patients, which develops following cutaneous manifestations. Only 5\% have symptoms such as abdominal pain, diarrhea, palpable mass, bleeding, obstruction or intestinal perforation. There is an increased risk of developing digestive malignancies, frequently in the small bowel. The following report presents the case of a patient with neurofibromatosis type 1, with the diagnosis of a jejunal gastrointestinal stromal tumor and a fibroid inflammatory polyp in the context of gastrointestinal bleeding.

Key words. Neurofibromatosis type 1, gastrointestinal stromal tumor, fibroid inflammatory polyp. 


\section{Abreviaturas}

NF-1: Neurofibromatosis tipo 1.

GIST: Tumor del estroma gastrointestinal.

PFI: Pólipo fibroide inflamatorio.

\section{Introducción}

La neurofibromatosis tipo 1 (NF-1), o enfermedad de von Recklinghausen, es un desorden hereditario autosómico dominante en el que se desarrollan tumores benignos y malignos en el sistema nervioso. ${ }^{1}$ La prevalencia de la enfermedad se estima entre 1 a 3000 y 1 en 6000 personas en el mundo. ${ }^{2}$ Se caracteriza por ser una enfermedad progresiva, multisistémica con penetrancia completa y expresividad variable. El defecto se encuentra en el gen supresor tumoral localizado en el cromosoma $17 \mathrm{q} 11.2$ que codifica la proteína neurofibromina. ${ }^{3}$ Se caracteriza por manchas color "café con leche", efélides axilares o inguinales, neurofibromas plexiformes, hamartomas del iris, displasia del ala del esfenoides, displasia de huesos largos, escoliosis y glioma óptico. ${ }^{4}$

La NF-1 se asocia con riesgo aumentado de malignidad. La implicancia de la neurofibromina en la vía RAS-MAPK interfiere con la proliferación y diferenciación celular y, por lo tanto, aumenta la predisposición al desarrollo de tumores malignos en estos pacientes. ${ }^{2}$ Dado el alto riesgo, la evaluación clínica de los pacientes debe ser realizada regularmente y ser referida a oncología cuando sea necesario.

En la NF-1, los tumores gastrointestinales ocurren con mayor frecuencia que los torácicos, endócrinos o la afectación arterial. ${ }^{5}$ El compromiso gastrointestinal se observa en $5-25 \%$, suele aparecer posteriormente a las manifestaciones cutáneas y sólo 5\% resulta sintomático con dolor abdominal, diarrea, masa palpable, sangrado, obstrucción o perforación intestinal. ${ }^{6,7}$

\section{Caso clínico}

Se presenta un paciente de sexo masculino, 53 años que consulta por anemia sintomática (astenia, adinamia y angor). Refiere episodios recurrentes de melena en forma intermitente en un periodo de aproximadamente 9 meses previos a la consulta actual. Como antecedentes patológicos se destacan: NF-1 diagnosticada en la adolescencia, acondroplasia, hipertensión arterial y cardiopatía isquémica.

$\mathrm{Al}$ examen físico presenta manchas "café con leche" en dorso y abdomen y múltiples nódulos cutáneos a predominio de dorso y región sacra compatibles con neurofibromas (Figura 1). Además presenta palidez cutáneo-
Figura 1. Lesiones cutánea en región sacra compatibles con neurofibromas

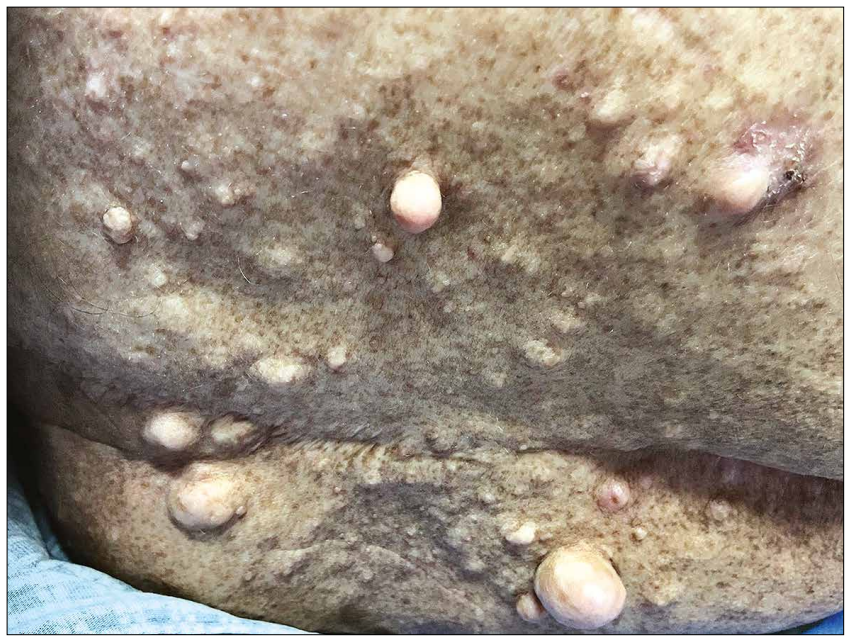

mucosa, taquicardia, queilitis angular. Al tacto rectal se constata melena.

El laboratorio confirma anemia con hematocrito 19,4\%; hemoglobina $6,5 \mathrm{~g} / \mathrm{dl}$; volumen corpuscular medio $87 \mathrm{fL}$. Posterior a su estabilización hemodinámica, que requiere múltiples transfusiones sanguíneas y valoración cardiológica para descartar isquemia aguda de miocardio, se solicitan los estudios endoscópicos.

La endoscopia alta se realiza explorando la región intestinal más distal posible, por la frecuencia de afectación intestinal, con los siguientes hallazgos: a nivel de cuarta porción duodenal y primera asa yeyunal una lesión polipoidea de 30-35 mm con superficie ulcerada. Se toman 3 biopsias profundas con la técnica de biopsia sobre biopsia del pólipo descrito. El estudio anatomopatológico informa una proliferación fusocelular positiva para CD117, DOG1 y CD34 por lo que se realiza el diagnóstico de tumor del estroma gastrointestinal (GIST).

La colonoscopia evidencia a nivel de ciego, en contacto con la válvula ileocecal, una lesión polipoidea pediculada de $40 \mathrm{~mm}$ con patrón glandular y vascular inespecífico (Figura 2). Se realiza inyección de adrenalina en el pedículo y se reseca con asa de diatermia en un fragmento. La anatomía patológica de dicha polipectomía informa un fragmento polipoideo erosionado con disminución de la mucosecreción de las glándulas y marcados cambios regenerativos. Se destaca en el sector central un componente fusocelular sin atipías, vasos ramificados y abundante exudado inflamatorio (Figura 3). La inmunohistoquímica evidencia positividad para CD34, fascina, calponina y ciclina D1. Esto apoya el diagnóstico de pólipo fibroide inflamatorio (PFI). 
Figura 2. Pólipo cecal. A: en ciego, en contacto con la válvula ileocecal, se observa lesión polipoidea pediculada de $40 \mathrm{~mm}$ con patrón glandular y vascular inespecífico. B: se realiza cromoendoscopía para verificar sus características con indigo carmin y FICE filtro 6
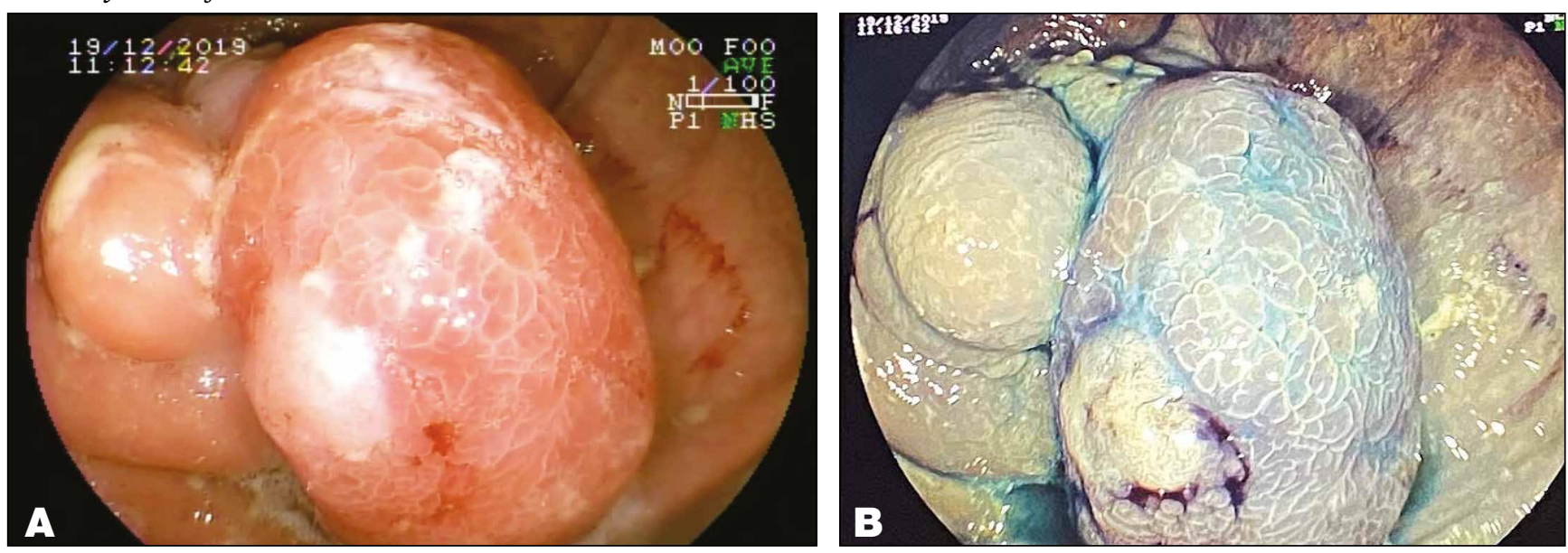

Se realiza tratamiento quirúrgico de la lesión yeyunal. La exploración laparoscópica evidencia una lesión exofítica de $30 \mathrm{~mm}$ en la segunda asa yeyunal. Se reseca por enucleación y se realiza enterotomía con anastomosis latero-lateral (Figura 4). La anatomía patológica de la pieza quirúrgica evidencia lesiones de expansión fuso-celular que comprometen la serosa y la submucosa con estroma en sectores colagenizado con fibras skenoides y calcificaciones distróficas, morfología epitelioide de 5\% con anticuerpos positivos para CD117, CD34, Vimentina, S100, DOG-1ik. Estos hallazgos son confirmatorios de GIST (Figura 5).

En la evolución el paciente, clínicamente estable, no reitera cuadros de hemorragia digestiva. En el seguimiento se realiza una enteroTC de abdomen que identifica material de sutura en yeyuno sin elementos de recidiva local, a nivel intestinal y en marco cólico no se evidencian engrosamientos parietales, ni áreas de realce patológico.
Figura 3. Histología con hematoxilina y eosina de pólipo cecal. En el sector central un componente fusocelular sin atipias, vasos ramificados y abundante exudado inflamatorio

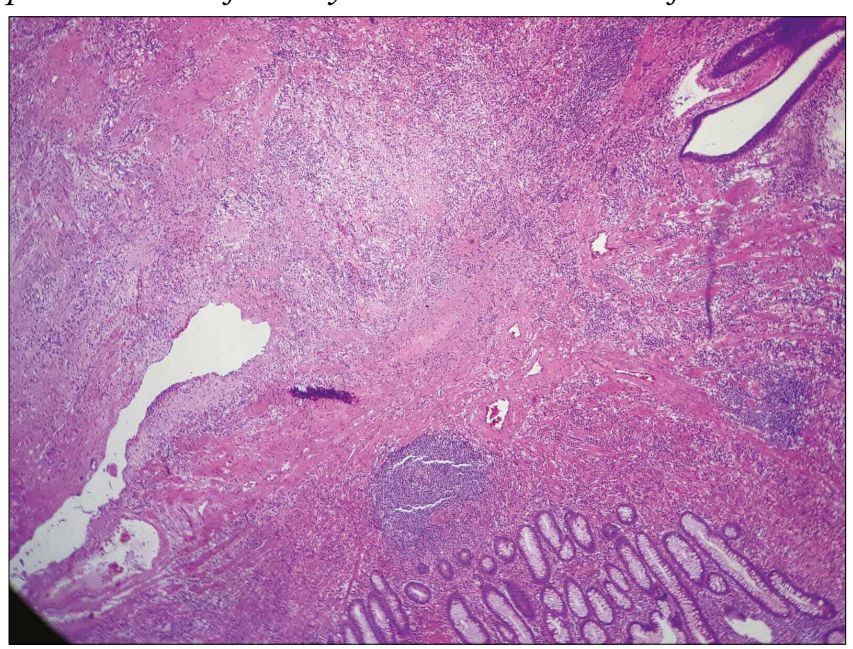

Figura 4. Pieza de resección quirúrgica de GIST intestinal

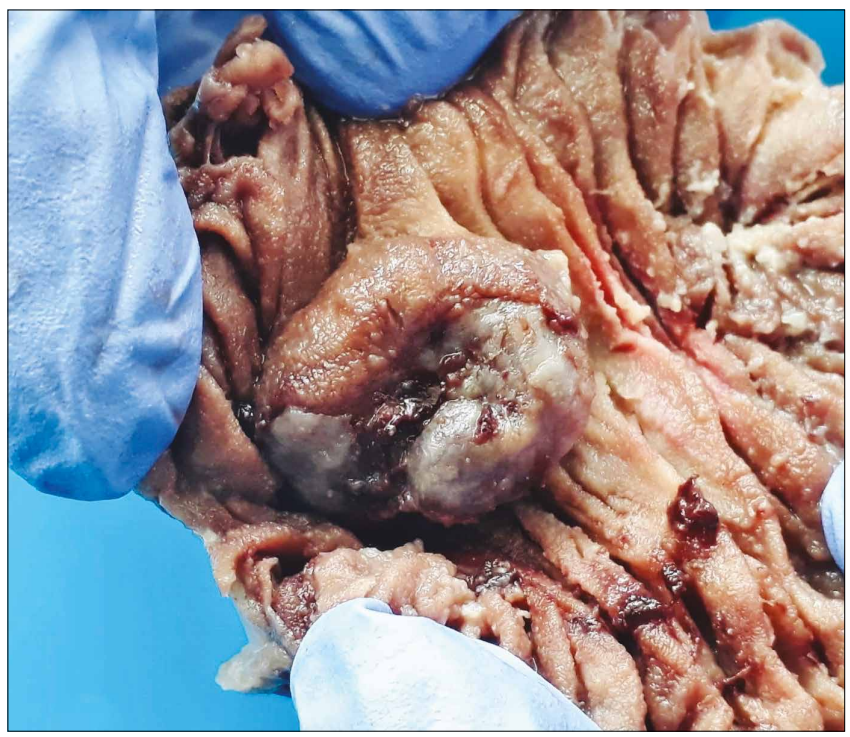


Figura 5. Histología con hematoxilina y eosina de GIST intestinal. Lesiones de expansión fuso-celular que comprometían la submucosa con estroma en sectores colagenizado con fibras skenoides y calcificaciones distróficas

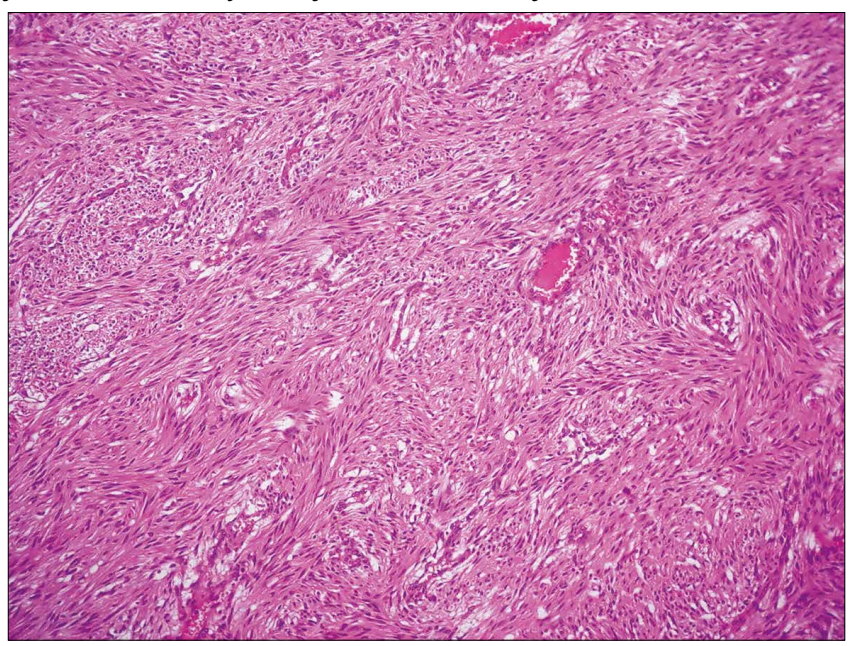

\section{Discusión}

Se presenta el caso de un paciente con el antecedente de NF-1 que en el estudio de una hemorragia digestiva se diagnostica un GIST yeyunal y una lesión polipoidea cecal la cual corresponde a un PFI. Los pacientes con NF-1 tienen riesgo incrementado de desarrollar tumores gastrointestinales, entre ellos GIST, con una incidencia de $6,5 \%$ por año. $^{8}$

El intervalo promedio desde el inicio de los síntomas hasta la detección de lesiones gastrointestinales en pacientes con NF-1 se realiza de forma tardía, según el reporte de una serie de casos (2,8 años). ${ }^{9}$ En este paciente transcurren 9 meses desde el comienzo de los síntomas. En pacientes con NF-1 existen diferentes formas de lesiones digestivas siendo las más relevantes la hiperplasia de la submucosa y de las células Schwann de los plexos nerviosos mioentéricos, formación de diferentes variedades de tumores estromales, mayor prevalencia de tumores carcinoides en la zona periampular y mayor predisposición que la población general a padecer adenocarcinomas intestinales (presentes en hasta 23\%). Los tumores gastrointestinales se ubican con más frecuentemente en intestino delgado (72\%). Las lesiones benignas diagnosticadas con mayor frecuencia son neurofibromas $52 \%$, leiomiomas $13 \%$, ganglioneurofibromas $9,8 \%$ y los GIST ocupan el cuarto lugar. ${ }^{9}$

En la NF-1, los GIST tienden a ocurrir a edades más tempranas, pero pueden presentarse de forma tardía. Tienen una ligera predominancia en mujeres. A menudo son múltiples y frecuentemente se encuentran en el intestino delgado: hasta $6 \%$ son duodenales y $4 \%$ yeyuno-ileales. ${ }^{10}$ Se considera que un tercio de las lesiones menores de $30 \mathrm{~mm}$ cursan sintomáticas. Los síntomas más frecuentes son dolor abdominal, obstrucción intestinal, sangrado y perforación. ${ }^{11}$

Si bien la técnica de biopsia sobre biopsia tiene bajo rendimiento al tratarse de lesiones submucosas, es posible realizar el diagnóstico como sucedió en este caso. En los hallazgos histológicos, estas lesiones muestran células fusiformes con eosinófilos PAS-positivos. Un aspecto importante en estos tumores es la ausencia de mutaciones del gen KIT y factor receptor alfa derivado de crecimiento de plaquetas (PDGFRA), típica de los GIST esporádicos, por lo que tiene pobre respuesta al tratamiento con inhibidores de la tirosina quinasa (Imatinib). Debido a esto, el tratamiento de los GIST asociados a NF-1 se basa en la resección endoscópica mediante enucleación o la resección quirúrgica completa. ${ }^{12}$

Si bien la prevalencia del compromiso gastrointestinal en la NF-1 es frecuente, la asociación de esta enfermedad con PFI, como en el caso presentado, ha sido poco descrita. ${ }^{13} \mathrm{El} \mathrm{PFI}$ es una lesión generalmente única y de aspecto polipoide que se localiza en la zona alta de la submucosa o la porción más profunda de la mucosa digestiva. Está constituido por una proliferación mal circunscrita de células fusiformes y fibras conjuntivas dispuestas concéntricamente alrededor de capilares y un grado variable de infiltrado inflamatorio con eosinófilos. ${ }^{14}$ La topografía más frecuente es a nivel de antro gástrico y yeyuno-íleon, si bien en algunos casos, como el de este paciente, se encuentran en colon. ${ }^{15}$ Afecta por igual a ambos sexos, con un pico de incidencia en la quinta $y$ séptima década. Al momento del diagnóstico la mayoría tienen un tamaño de entre 30 y $40 \mathrm{~mm} .{ }^{16,18}$ Si bien en este caso el PFI fue un hallazgo en la videocolonoscopía, se puede manifestar clínicamente tras su ulceración con hemorragia digestiva, anemia ferropénica o por obstrucción. ${ }^{14,19}$ No presenta potencial maligno y la recurrencia reportada es rara. ${ }^{16,17}$

En conclusión, en la NF-1, debido a la baja prevalencia, mayor afectación del intestino delgado y riesgo aumentado de neoplasias, se necesita el diagnóstico y tratamiento oportuno de las lesiones gastrointestinales. En este caso la hemorragia digestiva permitió realizar el diagnóstico de un GIST complicado, localizado a nivel yeyunal y un PFI en colon. Por este motivo, consideramos importante la exploración endoscópica profunda más allá de la segunda porción duodenal y con una correcta visualización ileal, así como también realizar una vigilancia adecuada de lesiones intestinales con potencial maligno. 
Conflicto de intereses. Los autores declaran no tener ningún conflicto de intereses.

\section{Sostén financiero. No se recibió apoyo financiero para la realización de este manuscrito.}

\section{Referencias}

1. Gómez-Zuleta MA, Lúquez-Mindiola AJ. Neurofibromatosis tipo 1 y sangrado de intestino delgado. Reporte de Caso. Rev Fac Med 2017; 65 (4): 683-685.

2. Correa M, Pasik N. Neurofibromatosis tipos 1 y 2, Revisión. Rev Hosp Ital BA 2019; 39 (4): 115-127.

3. Gottfried ON, Viskochil DH, Couldwell WT. Neurofibromatosis type 1 and tumorigenesis: molecular mechanisms and therapeutic implications. Neurosurg Focus 2010; 28 (1): E8.

4. Hirbe AC, Gutmann DH. Neurofibromatosis type 1: a multidisciplinary approach to care. Lancet Neurol 2014; 13 (8): 834-843.

5. Rastogi R. Intra-abdominal manifestations of von Recklinghausen's neurofibromatosis. Saudi J Gastroenterol 2008; 14 (2): 80-82.

6. Agaimy A, Vassos N, Croner RS. Gastrointestinal manifestations of neurofibromatosis type 1 (Recklinghausen's disease): Clinicopathological spectrum with pathogenetic considerations. Int J Clin Exp Pathol 2012; 5 (9): 852-862.

7. Ferner RE, Huson SM, Thomas N, Moss C, Willshaw H, Evans DG. Guidelines for the diagnosis and management of individuals with neurofibromatosis 1. J Med Genet 2007; 44 (2): 81-88.

8. Giuly JA, Picand R, Giuly D, Monges B, Nguyen-Cat R. Von Recklinghausen disease and gastrointestinal stromal tumors. Am J Surg 2003; 185: 86-87.

9. Rastogi R. Intra-abdominal manifestations of von Recklinghausen's neurofibromatosis. Saudi J Gastroenterol 2008; 14 (2): 80-82.
10. Toapanta L, Chable F, Tellez F. Neurofibromatosis y tumor estromal gastrointestinal diagnosticado por enteroscopia anterograda. El sevier endoscopia 2015; 27 (1): 45-48.

11. Saha SB, Parmar R, Mandal A. Small bowel obstruction in a neu-rofibromatosis patient-A rare presentation of gastro-intestinalstromal tumors (GISTs): Case report and literature review. Indian J Surg 2013; 75: 415-417.

12. Bakker JR, Haber MM, García FU. Gastrointestinal neurofibromatosis: an unusual cause of gastric outlet obstruction. Am Surg 2005; 71: 100-105.

13. Goto K, Hirosaki T, Masubuchi M. Neurofi-bromatosis Type 1 -associated inflammatory polyp of the gastrointestinal tract. Int J Surg Pathol 2017; 25: 65-68.

14. Acero D, Garijo G, Hombrados M, Figa M, Adrados M, Aldeguer X. Pólipo fibroide inflamatorio gastrointestinal. Características clínicas y seguimiento de una serie de 26 pacientes. Gastroenterol Hepatol 2005; 28 (4): 215-220.

15. Van de Rijn M, Hendrickson M, Rouse RV. An immunohisto-chemical study of inflammatory fibroid polyp of the gastrointestinal tract. Appl Immunohistochem 1994; 2: 54-59.

16. Bassam A.Vanek's tumor of the small bowel in adults. World J Gastroenterol 2015; 21 (16): 4802-4808.

17. Acero D, Garijo D, Hombrados M, Figa, Adrados M, Aldeguer X, González-Huix F, Miró J, Bernado L. Pólipo fibroide inflamatorio gastrointestinal. Características clínicas y seguimiento de una serie de 26 pacientes. Gastroenterol Hepatol 2005; 28 (4): 215-220.

18. Ng C, Lam KY, Gupta TS, Ho YH. Inflammatory Fibroid Polyp of the Caecum in a Patient with Neurofibromatosis. Ann Acad Med Singapure 2004; 33: 797-799.

19. Shimer GR, Helwig EB. Inflammatory fibroid polyps of the intestine. Am J Clin Pathol 1984; 81: 708-714. 